\title{
EPIDEMIOLOGIA
}

\section{Brote de shigelosis en una escuela de educación básica}

\author{
SOLEDAD CARRASCO L. ${ }^{1}$, M.V. VERONICA SOLARI G. ${ }^{2}$, VALERIA PRADO J. ${ }^{3}$, \\ LORNA SUAZO C. ${ }^{4}$, T.A. CAROLINA ARELLANO C. ${ }^{3}$, E.U. MARGARITA HERNANDEZ C. ${ }^{3}$ y \\ E.U. CECILIA ESPINOZA A. ${ }^{1}$
}

\section{SHIGELLA OUTBREAK IN AN ELEMENTARY SCHOOL}

In autumn 1996, children from an elementary school in Santiago were affected by an outbreak caused by Shigella sonnei. Initially 35 children out of 350 beneficiaries of the feeding program got sick. However, in the next five days, 189 new cases appeared. Sixty eight patients submitted stool samples for culture (65 children and 3 food handlers); $20.5 \%$ of stool cultures from the children were positive for $\boldsymbol{S}$. sonnei, and all samples from food handlers were negative. The presentation of the outbreak states a toxiinfection due to $S$. sonnei, which probably started by ingestion of contaminated food (rate of primary attack 10\%) and then person to person transmission (rate of secondary attack 16.9\%). To control the outbreak, the personal sanitation rules were enforced as well as those concerning the environment with educational lectures to all the school community and with sanitary in situ control. Five days after beginning the first case, trimethoprim-sulpha was administered to only those symptomatic cases during five days.

Key words: Outbreak; Diarrhea; Shigella sonnei; School children.

\section{INTRODUCCION}

De acuerdo a la experiencia latinoamericana, los patógenos más frecuentes causantes de diarrea aguda son, en orden de importancia relativa: Escherichia coli enteropatógena clásica, E. coli enterotoxigénica, rotavirus, Shigella spp, Campylobacter sp y Yersinia sp. ${ }^{1-}$

Las infecciones por Shigella son endémicas en esta región de las Américas, responsables de 8 a $12 \%$ del total de los episodios de diarrea y de 25 a $30 \%$ de los casos de diarrea con sangre. Cerca de $50 \%$ de los episodios de diarrea disentérica requieren hospitalización. ${ }^{6-8}$ Las especies de Shigella flexneri y Shigella sonnei son las más comunes en Chile con una proporción similar entre ambas, predominando $S$. sonnei cada dos a tres años. ${ }^{1,6}$

Estudios recientes realizados por C. Ferre-

Unidad de Epidemiología, Servicio de Salud Metropolitano Central.

2 Unidad de Epidemiología, Servicio de Salud Metropolitano del Ambiente.

3 Unidad de Microbiología-Oriente, ICBM, Facultad de Medicina, Universidad de Chile.

${ }_{4}$ Centro de Salud Dr. Norman Voullieme, Servicio de Salud Metropolitano Central. 
ccio et al en Santiago de Chile, ${ }^{6}$ han demostrado que la incidencia de Shigella es más elevada en niños de 1 a 4 años de edad como causa de diarrea (fue aislada en $10 \%$ de los episodios de diarrea ambulatoria y en $13,7 \%$ de los pacientes hospitalizados por diarrea, provenientes de la misma comunidad estudiada). La shigelosis presenta una clara estacionalidad, siendo más frecuentes en los meses de primavera y verano. ${ }^{1}$

La baja dosis infectante y la existencia de factores predisponentes, tales como hacinamiento y condiciones higiénicas deficitarias, tanto ambientales como personales, contribuyen a que el mecanismo de contagio más frecuente sea el fecal-oral. ${ }^{1,9}$

La principal forma de transmisión es persona a persona, especialmente en niños pequeños que asisten a jardines infantiles y en personas ancianas residentes en centros cerrados. En brotes de gastroenteritis, las fuentes de propagación son el agua y los alimentos contaminados, especialmente aquellos vegetales que se consumen crudos. ${ }^{10-20}$ El riesgo de contaminación de alimentos manipulados por individuos infectados y el antecedente de que puede aislarse Shigella en juguetes u otros objetos de uso común en centros de atención infantil -los cuales también constituyen vías de propagación de la infección- determinan que la aparición de casos de shigelosis en estos centros represente un problema de potencial importancia epidemiológica, si no se aplican medidas de prevención y control oportunas y adecuadas. ${ }^{21-}$ 23

Nuestro propósito es comunicar la experiencia de manejar un brote de diarrea aguda producida por $S$. sonnei en una escuela de educación básica.

\section{PACIENTES Y METODOS}

El brote se presentó en una escuela básica de la comuna de Cerrillos, en la ciudad de Santiago, en marzo de 1997. Este establecimiento imparte instrucción básica a 1.470 niños de nivel socioeconómico bajo, cuyas edades fluctúan entre 5 y 14 años. A la fecha señalada, 350 de sus alumnos se beneficiaban con el Programa de Alimentación Escolar, (PAE) por lo que almorzaban en la escuela.

A fines del verano de 1997, la Unidad de Epidemiología del S.S.M.C. recibió la notificación desde un servicio de emergencia, de la consulta de 35 niños que presentaban diarrea, dolor abdominal y vómitos, procedentes de esta escuela y con el antecedente común de haber ingerido el almuerzo escolar. Dos de los consultantes requirieron hospitalización por síndrome disentérico en el Hospital Clínico San Borja-Arriarán. Al momento de la primera consulta en emergencia, se tomó coprocultivo a los tres menores más afectados clínicamente, entre éstos, dos que fueron hospitalizados posteriormente.

Se definió como caso sospechoso de shigelosis, para fines operativos y cálculo de tasa de ataque primaria, a todos los alumnos de la escuela que almorzaron la ración del PAE el día 27 de marzo y que presentaron en el lapso de las 48 horas siguientes, un cuadro de vómitos y/o diarrea con o sin sangre, y/o dolor abdominal, con o sin fiebre y sin otra etiología conocida. Se incluyeron, además, los menores con coprocultivos positivos y cuyas muestras fueron tomadas en la unidad de emergencia el 27 de marzo.

Durante los 5 días siguientes se reportaron 189 nuevos casos procedentes de diferentes cursos de la misma escuela. Para el cálculo de la tasa de ataque secundaria se definió como caso a los alumnos que presentaron un cuadro similar sin otra etiología conocida, no beneficiarios del PAE y con asistencia regular las dos semanas posteriores al 27 de marzo. Se incluyó a todos los menores con coprocultivo positivo y cuyas muestras fueron tomadas en la escuela los primeros días tras el inicio del brote. Tan pronto se notificaron los primeros casos, se realizó una visita epidemiológica al establecimiento escolar. En ella se sugirieron correcciones a las deficiencias estructurales y sanitarias observadas y se tomaron muestras de agua para análisis microbiológico y de cloro residual. No existían restos alimentarios susceptibles de ser sometidos a un análisis microbiológico ya que se habían consumido o eliminado los alimentos de la colación sospechosa de estar contaminada. 
Al cuarto día de iniciado el brote, se tomó muestra de deposición mediante hisopado rectal, a 65 niños con diarrea y a los 3 manipuladores de alimentos del establecimiento. Estos manipuladores no presentaron diarrea antes, durante ni después de ocurridos los casos índices. Las muestras fueron transportadas en medio de Cary Blair al laboratorio de la Unidad de Microbiología-Oriente, del Programa de Microbiología de la Facultad de Medicina de la Universidad de Chile, donde fueron procesadas para identificar en el cultivo cinco categorías de E. coli diarreogénicas mediante hibridización de colonias con sondas genéticas (ECEP, ECET, ECEH, ECEAgg, ECEI) y para Salmonella, Shigella, Campylobacter y Yersinia, mediante técnicas microbiológicas tradicionales. Además se investigó la presencia de rotavirus y calicivirus con técnicas inmunoenzimáticas(ELISA) no comerciales.
Del mismo modo, se realizaron reuniones informativas de la situación y educativas, con énfasis en prevención de infecciones entéricas y técnica de lavado de manos al personal auxiliar, profesores, alumnos y apoderados.

A partir del quinto día de producidos los casos índices y una vez identificado el agente etiológico con su susceptibilidad antimicrobiana in vitro, se indicó la administración de cotrimoxazol en dosis habituales por espacio de 5 días, sólo a los niños sintomáticos. Se autorizó la reincorporación a clases una vez que cesaron los síntomas y signos de infección en cada paciente (Figura 1).

\section{RESULTADOS}

En este brote de gastroenteritis, la tasa de ataque primaria fue de $10 \%$, enfermando casi simultáneamente 35 de los 350 niños que al-

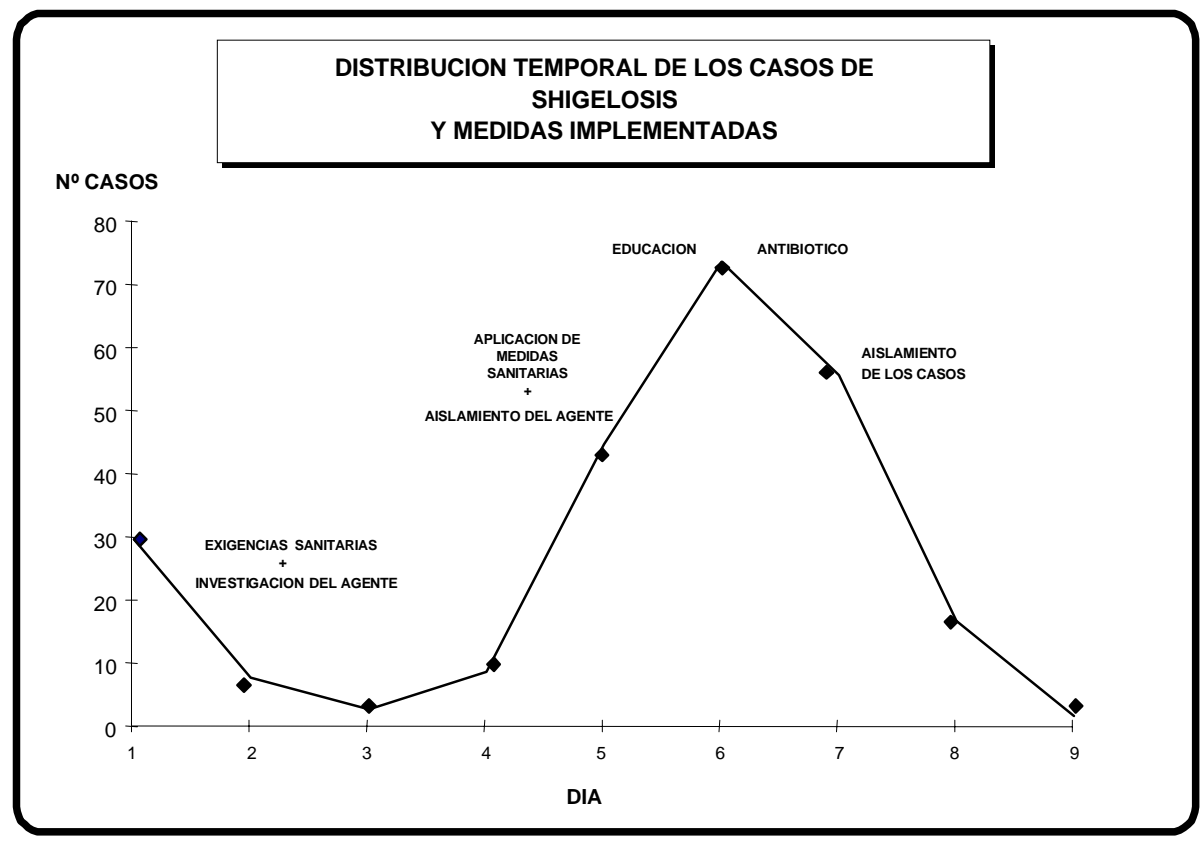

Figura 1. La figura muestra la relación temporal entre el número de casos afectados y las medidas de diagnóstico y control adoptadas. Durante los tres días siguientes a la aparición de los primeros casos, se realizó en terreno el diagnóstico epidemiológico de las personas y del ambiente, se sugirieron medidas sanitarias correctoras ambientales y se realizaron coprocultivos. A partir del quinto día, identificado el agente etiológico e implementadas las medidas sanitarias de control ambiental, se realizó educación sanitaria a toda la comunidad escolar, se administró antibióticos a los sintomáticos y se enfatizó en el aislamiento en el hogar de los casos. El brote se controló al noveno día de evolución. 
morzaban regularmente en el recinto. No fue posible identificar el alimento contaminado ni tampoco obtener tasas diferenciales ya que todos los niños consumieron la totalidad de la colación.

Posteriormente se inició la transmisión persona a persona, viéndose afectados en el lapso de 5 días, 189 de los restantes alumnos de la escuela (tasa de ataque secundario 16,9\%) (Tablas 1 y 2$)$.

Los casos recibieron atención médica en diferentes establecimientos y en la mayoría de ellos sólo se obtuvo el diagnóstico clínico. Aunque no en todos los pacientes hubo confirmación bacteriológica, los antecedentes epidemiológicos y el cuadro clínico sugieren una etiología común.

Del total de 68 coprocultivos realizados, 14 (20,5\%) resultaron positivos para S. sonnei, incluyendo a los dos menores hospitalizados. El antibiotipo fue similar para todas las cepas

Tabla 1. Características epidemiológicas de un brote de diarrea aguda en escuela básica

\begin{tabular}{lll}
\hline $\mathrm{N}^{\circ}$ total de alumnos & $=1.470$ \\
$\mathrm{~N}^{\circ}$ total de pacientes afectados & $=224$ \\
Edad promedio (rango de edad) & $=8$ años $(5-14$ años) \\
Tasa de ataque primario & $=10 \%(35 / 350)$ \\
Tasa de ataque secundario & $=16,9 \%(189 / 1.120)$ \\
Coprocultivo positivo & $=20,5 \%(14 / 68)$ \\
(Shigella sonnei) &
\end{tabular}

Tabla 2. Distribución por edad de los casos de diarrea en escuela básica

\begin{tabular}{ccr}
\hline $\begin{array}{l}\text { Edad } \\
\text { años }\end{array}$ & n & \% \\
\hline 5 & 15 & 6,7 \\
6 & 52 & 23,2 \\
7 & 42 & 18,8 \\
8 & 20 & 8,9 \\
9 & 32 & 14,3 \\
10 & 35 & 15,6 \\
11 & 23 & 10,3 \\
12 & 3 & 1,3 \\
14 & 2 & 0,9 \\
& & \\
Total & 224 & 100,0 \\
\hline
\end{tabular}

mostrando susceptibilidad para cotrimoxazol y furazolidona, y resistencia a ampicilina y cloranfenicol. En los coprocultivos realizados a los tres manipuladores de alimentos no se aislaron las bacterias enteropatógenas consideradas en el estudio microbiológico.

En ninguna de las muestras de deposición analizadas se detectó alguno de los virus entéricos investigados.

El análisis microbiológico del agua potable de la red de distribución de la escuela resultó adecuado y dentro de los estándares, al igual que la determinación de cloro residual.

El análisis de las características clínicas en una muestra del brote, a base de los antecedentes aportados por 68 menores afectados, permitió establecer que $100 \%$ de ellos presentó diarrea y/o dolor abdominal, 14,7\% presentó fiebre y sólo 2 casos $(2,9 \%)$ diarrea con sangre, siendo éstos los casos más severos y que requirieron hospitalización (Tabla 3 ).

\begin{tabular}{|c|c|c|}
\hline \multicolumn{3}{|c|}{$\begin{array}{l}\text { Tabla 3. Características clínicas de } \\
\text { pacientes de un brote de diarrea aguda en } \\
\text { escuela básica }(n=68)\end{array}$} \\
\hline & $\mathbf{n}$ & $\%$ \\
\hline Dolor abdominal & 68 & 100 \\
\hline Fiebre & 10 & 14,7 \\
\hline Diarrea acuosa & 68 & 100 \\
\hline Diarrea con sangre & 2 & 2,9 \\
\hline
\end{tabular}

\section{DISCUSION}

Las características de presentación de este brote permiten plantear que se inició como una toxiinfección por $S$. sonnei, debida probablemente a la ingestión de alimentos contaminados y luego, la infección fue transmitida de persona a persona a otro importante número de alumnos. Entre los alimentos que componían el menú del día en que ocurrieron los casos índices, el alimento más sospechoso fue una entrada de atún manipulada en la cocina de la escuela. Esta transmisión fue facilitada por la baja dosis infectante de este agente, por 
las deficientes condiciones sanitarias del establecimiento y por los escasos hábitos de higiene personal de los alumnos.

Este brote pudo documentarse y controlarse gracias a la intervención de un equipo multidisciplinario constituido por epidemiólogos de instituciones públicas junto a microbiólogos universitarios, con la colaboración del equipo de salud de un establecimiento de atención primaria, pero no existe la capacidad institucional, infraestructura ni coordinación, para enfrentar brotes de intoxicación alimentaria en forma eficiente y oportuna.

Respecto de la edad de los pacientes involucrados en este brote, es interesante comentar, que si bien en condiciones endémicas las infecciones por Shigella tienen una mayor incidencia en niños entre 12 y 47 meses, también niños de edades mayores pueden ser afectados ya que, como se observó en este brote, escolares entre 6 y 11 años de edad se contagiaron y presentaron síntomas. La proporción de niños escolares que tuvo deposiciones disentéricas (2/224) fue significativamente inferior a lo que se observa en niños bajo 5 años de edad, en los cuales 25 a $30 \%$ de las shigelosis se asocian a diarrea con sangre.

El adecuado manejo de este brote de shigelosis se debió al oportuno diagnóstico etiológico que permitió orientar medidas de control específicas, junto a la aplicación simultánea de programas educativos con énfasis en higiene ambiental, personal y de los alimentos -los que constituyeron un importante mecanismo de interrupción de la transmisión del microorganismo- observándose un cese del brote en forma rápida. No se efectuó coprocultivos de control.

El uso de antibióticos en todos los casos sintomáticos es una medida eficaz para el tratamiento del episodio clínico, en términos de acortar la duración de los síntomas, y también para interrumpir la cadena de transmisión. La cepa de $S$. sonnei involucrada en el brote era sensible a cotrimoxazol. Ello concuerda con lo observado por los autores en una red nacional de vigilancia de resistencia antimicrobiana, en la cual S. sonnei (n: 219 cepas) presentó sólo $21 \%$ de resistencia a cotrimoxazol, a diferencia de $S$. flexneri cuya resistencia a este anti- biótico es de $76 \% .^{25}$ Es importante enfatizar que no se utilizó quimioprofilaxis en los contactos asintomáticos.

Esta experiencia puso de manifiesto una falencia en la capacidad de responder adecuadamente en situaciones de brotes de gastroenteritis. A la vez motivó la planificación de un proyecto colaborativo (Servicio de Salud Metropolitano del Ambiente, Unidad de Microbiología-Oriente de la Facultad de Medicina y Servicios de Salud), con el objeto de participar conjuntamente en el manejo y control de futuros brotes de enfermedades de transmisión entérica, e implementar métodos de estudio etiológico que incluyan agentes virales, bacterianos y parasitarios, utilizando técnicas para el diagnóstico rápido de enteropatógenos en deposiciones y alimentos.

\section{RESUMEN}

Se analiza un brote de gastroenteritis por Shigella sonnei, ocurrido en una escuela básica de una comuna de Santiago, en marzo de 1997. En esta escuela, con una matrícula de 1.470 niños entre 5 y 14 años de edad, 350 se beneficiaban con el Programa de Alimentación Escolar (PAE) y almorzaban en el establecimiento. Treinta y cinco de los 350 niños del PAE presentaron simultáneamente gastroenteritis que motivó consulta en un servicio de emergencia, 2 de los cuales presentaron diarrea con sangre requiriendo hospitalización. Durante los cinco días posteriores se registraron 189 nuevos casos en la escuela.

Frente a la notificación, se efectuó visita epidemiológica a la escuela, obteniéndose muestras de deposición para estudio de bacterias y virus enteropatógenos en 65 niños sintomáticos y en los tres manipuladores de alimentos. Se identificó S. sonnei en $20,5 \%$ de los coprocultivos y todas las cepas tenían el mismo antibiotipo. La búsqueda de virus entéricos (rotavirus, calicivirus) dio resultados negativos. En los manipuladores de alimentos no se detectó enteropatógenos bacterianos ni virales.

La presentación del brote plantea una toxiinfección por $S$. sonnei, iniciada probablemente por ingestión de alimentos contamina- 
dos (tasa de ataque primario $10 \%$ ) y luego transmisión persona a persona (tasa de ataque secundario $16,9 \%$ ).

Para controlar el brote se reforzaron medidas de higiene personal y de saneamiento ambiental a través de educación a toda la comunidad escolar y el control sanitario del establecimiento. A partir del quinto día de iniciado el primer caso, se administró cotrimoxazol, durante cinco días, sólo a los casos sintomáticos. El brote se controló al noveno día.

\section{BIBLIOGRAFIA}

1.- PRADO V, O'RYAN M. Acute gastroenteritis in Latin America. Infect Dis Clin North Am 1994; 8: 77-105.

2.- CRAVIOTO A, REYES R, ORTEGA R, FERNANDEZ G, HERNANDEZ R, LOPEZ D. Prospective study of diarrhoeal disease in a cohort of rural Mexican children: Incidence and isolated pathogens during de first two years of life. Epidemiol Infect 1988; 101: 123-34.

3.- LANATA C, BLACK R, MAURTUA D et al. Etiologic agents in acute versus persistent diarrhea in children under three years of age in periurban Lima, Perú. Acta Paediatr Scand Suppl 1992; 381: 32-8.

4.- LEVINE M, FERRECCIO C, PRADO V et al. Epidemiologic studies of Escherichia coli diarrheal infections in a low socieconomic level periurban community in Santiago, Chile. Am J Epidemiol 1993; 128: 849-69.

5.- GOMES T A, RASSI V, MACDONALD K et al. Enteropathogens associated with acute diarrheal disease in urban infants in Sao Paulo, Brazil. J. Infect Dis 1991; 164: 331-7.

6.- FERRECCIO C, PRADO V, OJEDA A et al. Epidemiologic patterns of acute diarrhea and endemic Shigella infections in children in a poor periurban setting in Santiago, Chile. Am J Epidemiol 1991; 134: 614-27.

7.- PRADO V, SIRI M, AVENDAÑO L et al. Prevalencia de agentes enteropatógenos en síndrome diarreico agudo en niños hospitalizados y ambulatorios. Rev Chil Pediatr 1987; 58: 285-90.

8.- BOEHME C, RODRIGUEZ G, ILLESCA V, REYDET P, SERRA J. Shigellosis in children of the IX Region of Chile: clinical and epidemiologic aspects and antibiotic sensitivity. Rev Méd Chil 1992; 120: 1261-6.

9.- ROSENBERG T, KENDALL O, BLANCHARD J, MARTEL S, WAKELIN C, FAST M. Shigellosis on Indian reserves in Manitoba, Canada: Its relationship to crowded housing, lack of running water, and inadequate sewage disposal. Am J Public
Health 1997; 87: 1547-51.

10.- KOLAVIC S, KIMURA A, SIMONS S, SLUTSKER L, BARTH S, HALEY C. An outbreak of Shigella dysenteriae type 2 among laboratory workers due to intentional food contaminations JAMA 1997; 278: 396-8.

11.- DUNN R, HALL W, ALTAMIRANO J, DIETRICH S, ROBINSON-DUNN B, JOHNSON D. Outbreak of Shigella flexneri linked to salad prepared at a central commissary in Michigan. Public Health Rep 1995; 110: 580-6.

12.- GESSNER-B, BELLES M. Moose soup shigellosis in Alaska. West J Med 1994; 160: 430-3.

13.- KEENE W, MC ANULTY J, HOESLY $F$ et al. A swimming-associated outbreak of hemorrhagic colitis caused by Escherichia coli $0157: \mathrm{H} 7$ and Shigella sonnei. NEJM 1994; 331: 579-89.

14.- HEDBERG C, LEVINE W, WHITE K et al. An international foodborne outbreak of shigellosis associated with a commercial airline. JAMA 1992; 268: 3208-12.

15.- BLOSTEIN J. Shigellosis from swimming in a park pond in Michigan. Public Health Rep 1991; 106: 317-22.

16.- PELLETIER A, FINGER R, SOSIN D. Shigellosis in Kentucky, 1986 through 1989. South Med J 1991; 84: 818-21.

17.- KAPPERUD G, RORVIK L, HASSELTVEDT V et al. Outbreak of Shigella sonnei infection traced to imported iceberg lettuce. J Clin Microbiol 1995; 33: 609-14.

18.- SAMONIS G, ELTING L, SKOULIKA E; MARAKI S, TSELENTIS Y. An outbreak of diarrhoeal disease attributed to Shigella sonnei. Epidemiol Infect 1994; 112: 235-45.

19.- LEW J, SWERDLOW D, DANCE M et al. An outbreak of shigellosis aboard a cruise ship caused by a multiple-antibiotic-resistant strain of Shigella flexneri. Am J Epidemiol 1991; 134: 413-20.

20.- EGOZ N, SHMILOVITZ M, KRETZER B, LUCIAN M, PORAT V, RAZ R. An outbreak of Shigella sonnei infection due to contamination of a municipal water supply in northern Israel. J Infect 1991; 22: 87-93.

21.- MOHLE-BOETANI J, STAPLETON M, FINGER $\mathrm{R}$, BEAN $\mathrm{N}$, POUNDSTONE J, BLAKE P. Community wide shigellosis: control of an outbreak and risk factors in child day-care centers. Amer J Public Health 1995; 85: 812-6.

22.- MAHONEY F, FARLEY T, BURBANK D, LESLIE N, MC FARLAND L. Evaluation of an intervention program for the control of an outbreak of shigellosis among institutionalized persons. J Infect Dis 1993; 168: 1177-80.

23.- BRIAN M, VAN R, TOWNSEND L, MURRAY B, CLEARY T, PICKERING L. Evaluation of the molecular epidemiology of an outbreak of multiply resistant Shigella sonnei in a day-care center by using pulsed-field gel electrophoresis and plasmid DNA analysis. J Clin Microbiol 1993; 31: 2152-6. 
24.- PRADO V, LAGOS R, NATARO J. Populationbased study of the incidence of Shigella diarrhea and causative serotypes in Santiago, Chile. Pediatr Infect Dis J 1999; 18: 500-5.
25.- PIDAL P, PRADO V, TRUCCO O et al. Panorama de la resistencia antimicrobiana de Shigella $s p$. en 10 hospitales chilenos. Proyecto Pronares Rev Panam Infectol 1999; 1: S18-S25.

Correspondencia a:

Soledad Carrasco L.

Unidad de Epidemiologia SSMC

Av. V. Mackenna 477, tercer piso

Santiago, Chile

Fax: 56 (2) 6659397

Email: scarras@ssmc.cl 